\title{
Early multiplexed detection of cirrhosis by giant magnetoresistive biosensors with protein biomarkers
}

\author{
Elaine $\mathrm{Ng}^{\mathrm{a}, \mathrm{b}^{*}}$, An K. Le ${ }^{\mathrm{c}}$, Mindie H. Nguyen ${ }^{\mathrm{c}}$, and Shan X. Wang ${ }^{\mathrm{b}, \mathrm{d}}$ \\ a Department of Bioengineering, Stanford University, Stanford, CA, USA \\ ${ }^{\mathrm{b}}$ Department of Materials Science and Engineering, Stanford, CA, USA \\ c Division of Gastroenterology and Hepatology, Stanford University Medical Center, Palo Alto, CA, USA \\ d Department of Electrical Engineering, Stanford University, Stanford, CA, USA \\ "Corresponding author: Elaine Ng, Tel: (512) 577-2569, Email: elaineng@stanford.edu
}




\section{Standard Curves and Cross-reactivity Analysis}

Prior to running GMR-based immunoassays on clinical samples, the sensitivity and specificity of the platform and assays were characterized. To characterize the sensitivity and linear dynamic range of the multiplexed assay, standard curves with commercially available standard proteins were generated (sICAM-1, IL-6, TNF- $\alpha$, and CRP). Briefly capture probes were spotted on the sensor chip and blocked at room temperature for $1 \mathrm{hr}$ with 1\% BSA. A concentrated starting sample cocktail of standard proteins were made at a concentration of $10,000 \mathrm{pg} / \mathrm{mL}$. The sample cocktail was then diluted to lower concentrations $(5,000,1,000,500,100,50,25,10 \mathrm{pg} / \mathrm{mL})$. One sensor chip was designated the "blank" chip, where only wash buffer and no sample was added in order to determine the biological background noise signal. The sample cocktails of various dilutions were added to the sensor chips and incubated at room temperature for $1 \mathrm{hr}$, after which a cocktail of detection antibodies was added and incubated at room temperature for $1 \mathrm{hr}$. Chips were plugged into the GMR reader station, magnetic nanoparticles were added, and data was continuously collected until signals saturated at around $15 \mathrm{~min}$.

Saturated signals at the 15 min time point were used to generate the standard curves shown in Figure S1. Lower limits of detection were determined by taking the saturated signal values obtained from the "blank" chip and adding two standard deviations. Assays where commercially available standard proteins were available (sICAM-1, IL-6, TNF- $\alpha$, and CRP; R\&D Systems) show good linear dynamic range, about four orders of magnitude, and decent lower limits of detection. As expected, assays where commercially available standard proteins were not available (M2BPGi, NF-kB) did not generate a standard curve and are therefore not displayed in Figure S1. It should be noted that testing results for these two proteins are therefore solely based on clinical evaluations and would need additional evaluation for more clinical conclusions, especially given the small negative sample size. Table S1 shows the lower limits of detection of the 
multiplexed GMR-based immunoassays compared to those of the commercially available sandwich enzyme linked immunosorbent assay kits (R\&D Systems).

SICAM-1

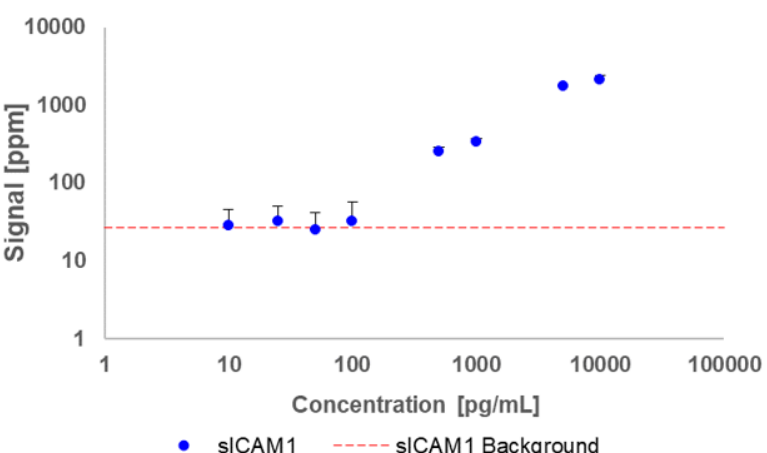

\section{IL-6}

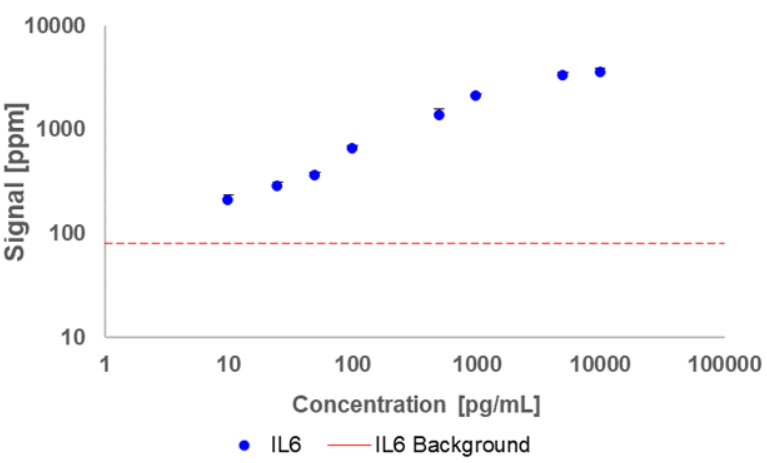

CRP

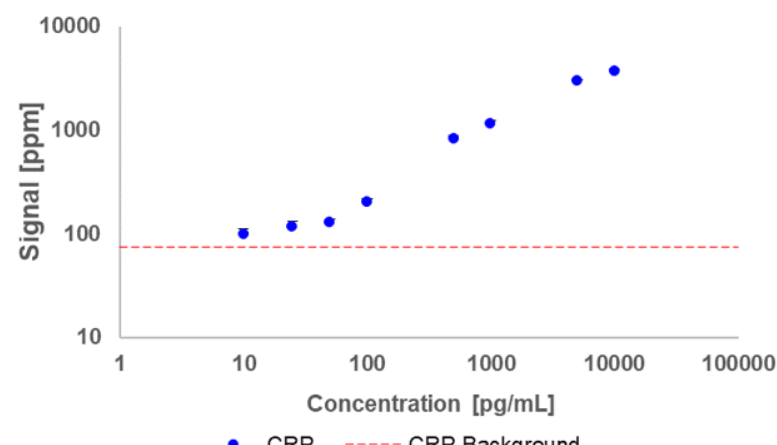

- CRP ---- CRP Background

\section{TNFa}

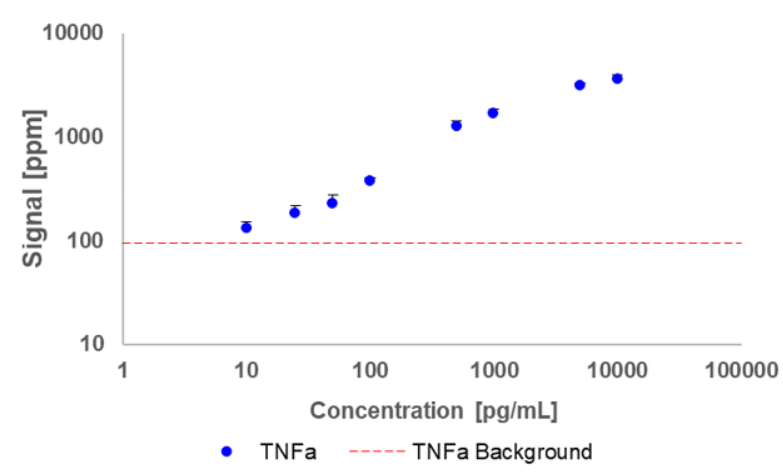

Figure S1. Standard curves of GMR-based Multiplexed Cirrhosis Protein Biomarker Panel Assay. Standard curves for the assays on the multiplexed cirrhosis protein biomarker panel were generated to characterize the sensitivity and linear dynamic range of the GMR multiplexed assay. Red dashed lines indicate the lower limit of detection. 


\begin{tabular}{ccc} 
Biomarker & $\begin{array}{c}\text { Multiplexed } \\
\text { GMR-based Assay LOD } \\
{[\mathrm{pg} / \mathbf{m L}]}\end{array}$ & $\begin{array}{c}\text { Commercially Available } \\
\text { Sandwich ELISA LOD } \\
{[\mathbf{p g} / \mathbf{m L}]}\end{array}$ \\
\hline SICAM-1 & 96.9 & 78.1 \\
IL-6 & 4.9 & 9.4 \\
TNF- $\alpha$ & 12.6 & 15.6 \\
CRP & 14.2 & 15.6
\end{tabular}

Table S1. Limits of detection (LOD) of the multiplexed GMR immunoassay compared to those of the commercially available sandwich ELISA kits (R\&D Systems).

Cross-reactivity analysis must be conducted to evaluate the specificity of the capture-detection probe pairs and to ensure that none of the reagents in the cirrhosis biomarker panel cross-react with each other to generate false positive signals. This is especially important to analyze in a multiplexed biomarker panel. Cross-reactivity analysis was performed using the method detailed by Gaster et al. Briefly, a single chip was spotted with all the promising capture probes that were intended to be used in the biomarker panel. Sensors were blocked with $1 \%$ BSA to prevent nonspecific binding. A cocktail sample of highly concentrated standard proteins in wash buffer was added to the sensors and incubated for $1 \mathrm{hr}$ at room temperature. After incubation, the sample was removed by aspiration and the sensor chip was plugged into the GMR reader station. Magnetic nanoparticles $(50 \mu \mathrm{L})$ were added to the sensor chip. One by one, individual biotinylated detection antibodies specific to the analytes of interest were pipetted onto the sensor surface at about 5 min intervals. As each detection antibody was added, only signals associated with the corresponding analyte were expected to rise. For example, when sICAM-1 detection antibody is added, only sensors that were spotted with sICAM-1 capture antibody, and therefore have formed a sICAM-1 sandwich immunoassay structure, should generate signals and binding curves. If no signals were generated, either the particular analyte was not included in the cocktail mixture, or the capture and/or the detection antibody were inadequate in detecting the particular analyte of 
interest. If multiple signals were generated, then there was a cross-reaction between the added detection antibody and the incorrect analyte or capture probe.

Figure $\mathbf{S 2}$ shows the result of the cross-reactivity analysis of the cirrhosis biomarker panel. Here, we used a sample cocktail consisting of sICAM-1, IL-6, TNF- $\alpha$, and CRP standard proteins that were commercially available (R\&D Systems). Standard proteins for M2BPGi and NF-KB were not available and therefore the specificity for these proteins could not be truly evaluated. However, the capture probes and detection antibodies were still evaluated for cross-reactivity with other proteins and reagents. As shown in Figure S2, sensors dedicated to the negative control BSA, M2BPGi, NF-kB did not generate any signals upon addition of individual detection antibodies, which was expected. This also suggests that the capture probes for M2BPGi and NF-KB do not cross-react with other assay reagents and their own respective detection antibodies. As expected, upon the addition of SICAM-1, IL-6, TNF- $\alpha$, and CRP detection antibodies, the sensors functionalized with the respective capture probes generated a single binding curve, suggesting that the added detection antibody bound specifically to the respective capture probe-analyte structure already on the sensor surface. However, as shown in Figure S2, upon adding TNF- $\alpha$ detection antibody to the chip, sensors functionalized with IL-6 CBL2117 capture probe generated a binding curve, in addition to the expected TNF- $\alpha$ binding curve. This suggests that the TNF- $\alpha$ detection antibody binds nonspecifically to the IL-6 CBL2117 capture probe. It also suggests that the TNF- $\alpha$ detection antibody does not nonspecifically bind to the IL- 6 protein itself. If this was the case, then the sensors functionalized with IL-6 13A5 would also generate a binding curve upon addition of the TNF- $\alpha$ detection antibody. As a result, the IL-6 13 A5 capture probe was preferentially kept in the cirrhosis biomarker panel, and the IL-6 CBL2117 capture probe was removed. All reagent pairs in the cirrhosis biomarker panel assay have therefore been verified for working viability and specificity. 


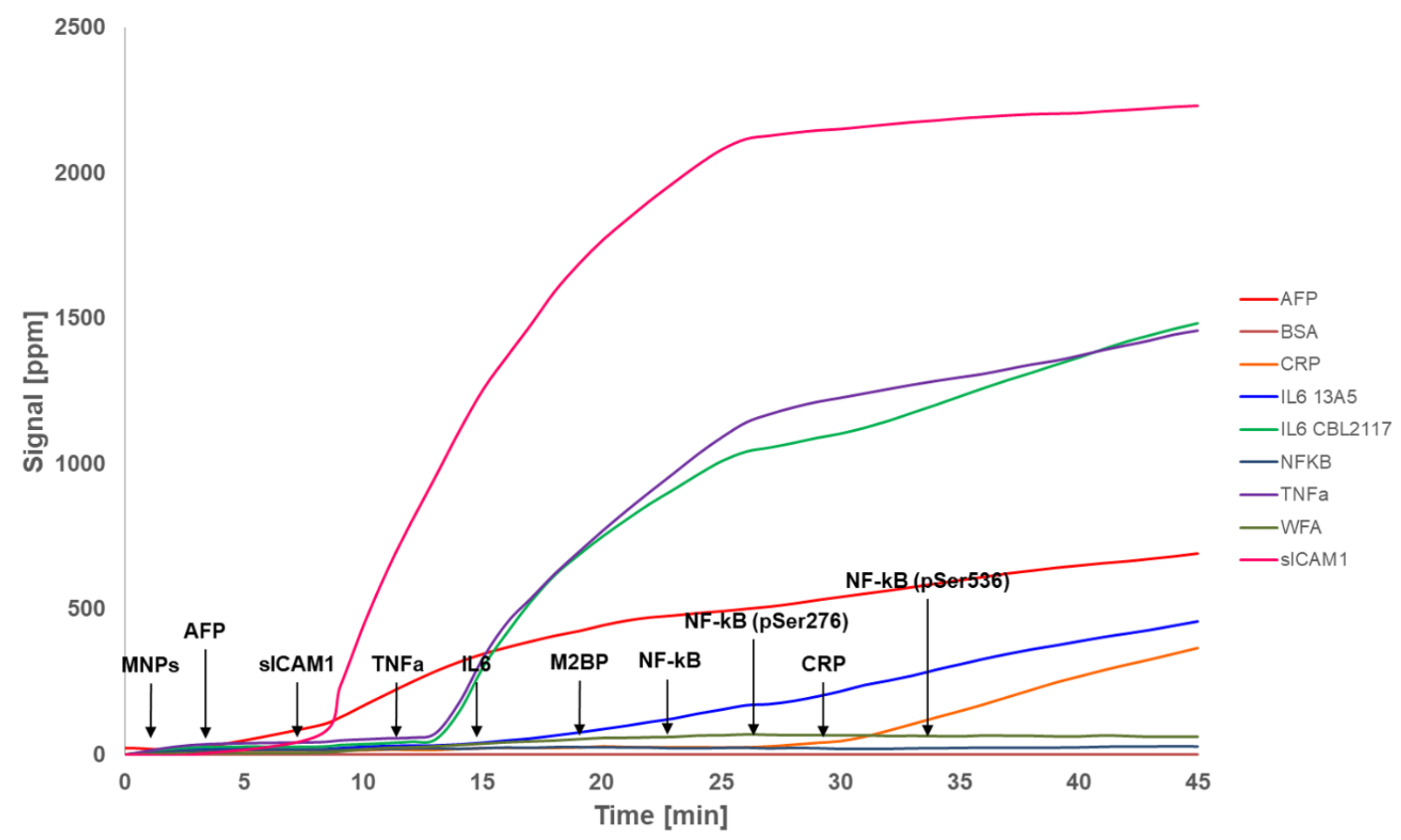

Figure S2. Cross-reactivity Evaluation of GMR-based Multiplexed Cirrhosis Protein Biomarker Panel Assay. Cross-reactivity amongst capture probe and detection antibody pairs were tested to evaluate the specificity of the multiplexed GMR-based cirrhosis biomarker panel assay. Labeled arrows indicate the time point at which a specific detection antibody was added. Signals from sensors dedicated to the various capture probes on the chip are represented by different colors in the legend. Upon addition of a specific detection antibody, a single signal is expected to appear: the binding curve associated with the analyte of interest. Any other stray signals present represent non-specific binding of the antibody to other proteins, and thus cross-reaction.

\section{Characterization of the Double-Use Immunoassay Method}

In order to test the cirrhosis protein biomarker panel in clinical patient plasma samples, CRP was detected in 5000x diluted plasma, low abundance biomarkers were detected in 5x diluted plasma, and intermediate abundance biomarkers were detected in 200x diluted plasma. To minimize the number of chips used while testing all the biomarkers, a double-use immunoassay method was implemented. Plasma was diluted by 5000x and added to the sensor chip for the GMR-based CRP immunoassay. After gathering GMR signals from the reader station and allowing CRP signals to saturate at $15 \mathrm{~min}$, the sensor chip was unplugged from the reader station and thoroughly washed using wash buffer to remove any unbound magnetic nanoparticles. Plasma 
that was diluted by $5 \mathrm{x}$ was then added to the washed sensor chip for the detection of low abundance biomarkers. A second chip was used to detect the intermediate biomarkers (sICAM1 and M2BPGi) in 200x diluted plasma, and therefore only utilized the normal single-use immunoassay method. In order to ensure that the assay signals were not strongly affected by this double-use immunoassay method, characterization of the method was performed.

Shown in Figure S3 are GMR signals for each of the low abundance biomarkers after a doubleuse immunoassay experiment, with the exception of chip 4, where a single-use immunoassay was performed to determine baseline GMR signals for the biomarkers at 50x diluted plasma. For chip 1,500x diluted plasma was used to detect CRP, followed by 50x diluted plasma to detect low abundance biomarkers. For chip 2, 1000x diluted plasma was used to detect CRP, followed by $50 x$ diluted plasma to detect low abundance biomarkers. For chip 3, 5000x diluted plasma was used to detect CRP, followed by $5 x$ diluted plasma to detect low abundance biomarkers.

When comparing GMR signals gathered from chip 1 to baseline GMR signals gathered from chip 4, all biomarker signals are comparable, and the double-use immunoassay method with 500x diluted plasma did not affect low abundance biomarker signal levels. However, in order to detect CRP within the linear dynamic range of the GMR immunoassay, plasma samples must be diluted by between $1000 x$ and $5000 x$. GMR signals from chip 2 show that there is an appreciable drop in low abundance biomarker signals after detecting CRP in 1000x diluted plasma. Therefore, a significantly lower dilution factor (such as $5 \mathrm{x}$ ) is needed to bring low abundance biomarker signals back to detectable ranges. GMR signals from chip 3 show that low abundance biomarker signals with $5 x$ diluted plasma, after detection of CRP in 5000x diluted plasma, are appreciably higher than the baseline signals from chip 4 with 50x diluted plasma and single-use method.

From these experiments, it can be seen that resulting signals for the low abundance biomarkers are a result of an accumulative effect from both high and low abundance biomarker tests. Chip 1 has higher low abundance biomarker signals than Chip 2 because the high abundance biomarker 
assay was performed at a lower dilution (500x versus $1000 x)$. Therefore, higher amounts of low abundance biomarkers were captured on Chip1 than on Chip 2, resulting in higher signals. It can also be seen that higher dilutions of plasma for CRP detection can affect downstream signals of low abundance biomarkers. However, this can be corrected by detecting low abundance biomarkers at lower dilutions of plasma. Therefore, when using the double-use immunoassay method to assay clinical plasma samples, 5000x diluted sample was used to detect CRP, followed by $5 x$ diluted sample was used to detect low abundance biomarkers on a single GMR chip. Intermediate abundance biomarkers were detected on a separate GMR chip in 200x diluted plasma.

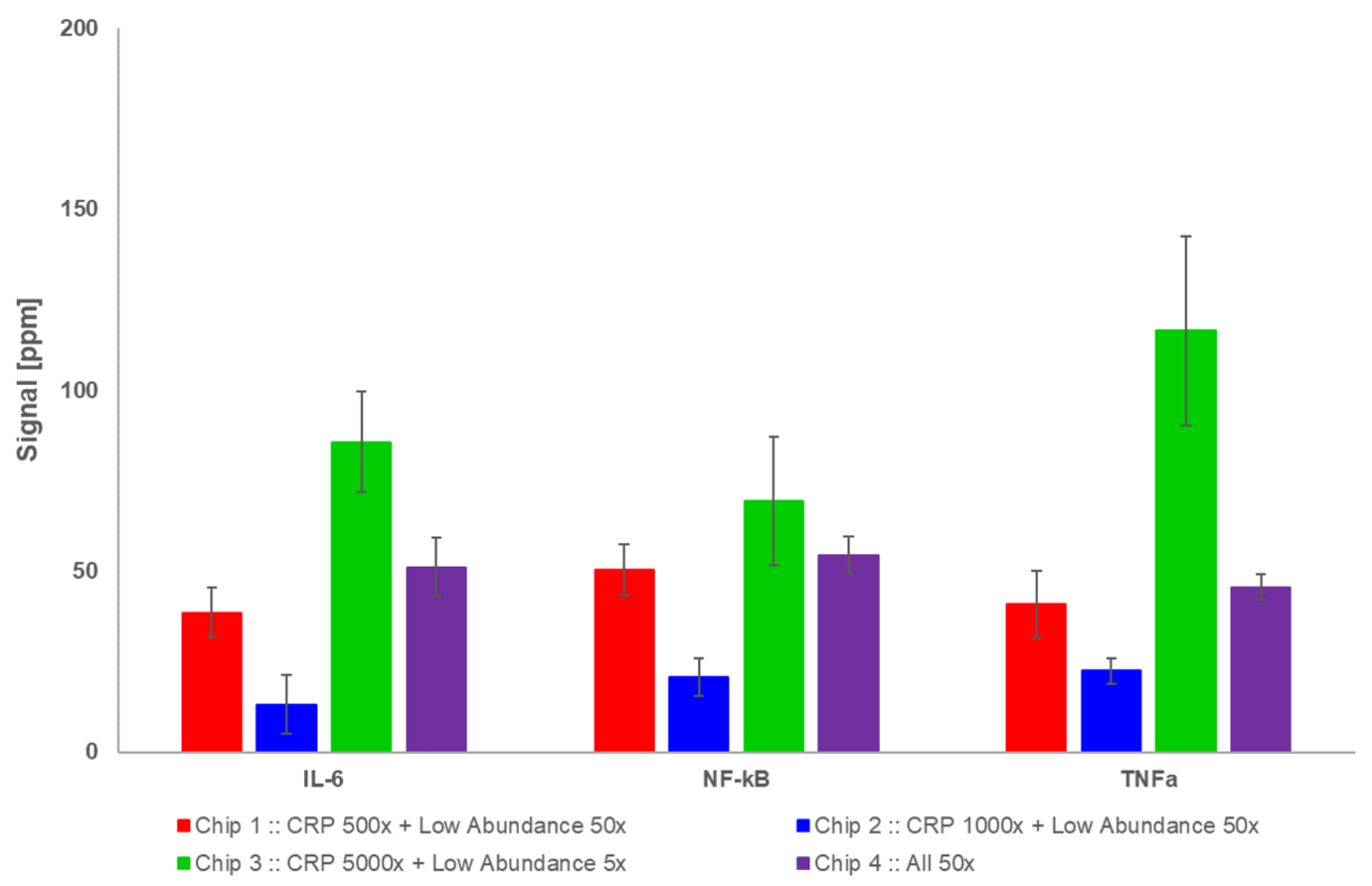

Figure S3. Double-Use Immunoassay Experiments. Various double-use immunoassay experiments (chips 1,2, and 3) with various plasma dilution factors were performed and GMR signals for low abundance biomarkers were compared to baseline signals from the normal single-use immunoassay method (chip 4). 